\title{
Histopathologically confirmed very late stent thrombosis associated with stent fracture after implantation of first-generation drug eluting stent
}

\author{
Takao Konishi ${ }^{1}$, Naohiro Funayama ${ }^{2}$, Tadashi Yamamoto ${ }^{2}$, Daisuke Hotta ${ }^{2}$, \\ Yuta Kobayashi ${ }^{1}$, Hirokazu Komoriyama ${ }^{1}$, Yoshiya Kato ${ }^{1}$, Kazunori Omote ${ }^{1}$, \\ Takuma Sato $^{1}$, Kiwamu Kamiya ${ }^{1}$, Toshiyuki Nagai ${ }^{1}$, Shinya Tanaka ${ }^{3}$, Toshihisa Anzai ${ }^{1}$ \\ ${ }^{1}$ Department of Cardiovascular Medicine, Faculty of Medicine and Graduate School of Medicine, \\ Hokkaido University, Sapporo, Japan \\ ${ }^{2}$ Department of Cardiology, Hokkaido Cardiovascular Hospital, Sapporo, Japan \\ ${ }^{3}$ Department of Cancer Pathology, Faculty of Medicine, Hokkaido University, Sapporo, Japan
}

A 76-year-old woman was admitted to hospital due to ST-segment elevation myocardial infarction with $3 \mathrm{~h}$ of evolution. She had undergone implantation of a first-generation sirolimus-eluting stent (SES) 8 years before in the mid left anterior descending artery (segment 7). Antiplatelet therapy was discontinued for 2 weeks because she had had surgical treatment for colon cancer. Emergency coronary angiography revealed a total occlusion of the implanted SES (Fig. 1A, arrow; Suppl. Video 1) Aspiration thrombectomy was performed and balloon dilatation achieved grade 3 Thrombolysis in Myocardial Infarction flow in a final coronary angiogram (Fig. 1B). The magnification image by stent boost revealed stent fracture (SF) of the SES (Fig. 1C, arrow). Histopathological examination revealed the presence of multinucleated giant cells (Fig. 1D, arrows) with many macrophages in the aspirated thrombi, suggesting a foreign body reaction to the implanted SES. The patch test for metal allergy was negative. These findings suggested that SF and discontinuation of antiplatelet therapy were the most likely causes of very late stent thrombosis, rather than a metal allergy. The presence of SF is a risk factor for stent restenosis and thrombosis. Delayed hypersensitivity reactions involving eosinophilic infiltrates have been previously described in first-generation drug-eluting stents [1]. The presence of macrophages and giant cells surrounding fragments of polymer strongly suggested a role of the latter in triggering a delayed hypersensitivity and subsequent thrombosis [1]. Very late stent thrombosis due to other mechanisms (e.g. altered biomechanics in the vessel) is however associated to different histopathological infiltrates [2]. According to available research, this is the first case to report macrophagic/giant cell infiltration without eosinophils in a thrombosis of first-generation SES due to stent fracture. The patient was discharged asymptomatic under antiplatelet therapy for secondary prevention.

Conflict of interest: None declared

\section{References}

1. Virmani R, Guagliumi G, Farb A, et al. Localized hypersensitivity and late coronary thrombosis secondary to a sirolimus-eluting stent: should we be cautious? Circulation. 2004; 109(6): 701-705, doi: 10.1161/01.CIR.0000116202.41966.D4, indexed in Pubmed: 14744976 .

2. Jaguszewski MJ, Cortes C, Daucher H, et al. Very late stent thrombosis in everolimus-eluting stent with predisposing mechanical factors: Differential features. Cardiol J. 2017; 24(4): 345-349, doi: 10.5603/CJ.2017.0089, indexed in Pubmed: 28831778.

Address for correspondence: Takao Konishi, MD, PhD, Department of Cardiovascular Medicine, Faculty of Medicine and Graduate School of Medicine, Hokkaido University, West 7, North 15, Kita-ku, Sapporo, 060-8638, Japan, e-mail: takaokonishi0915@gmail.com

Received: 26.01.2020 Accepted: 11.02.2020 


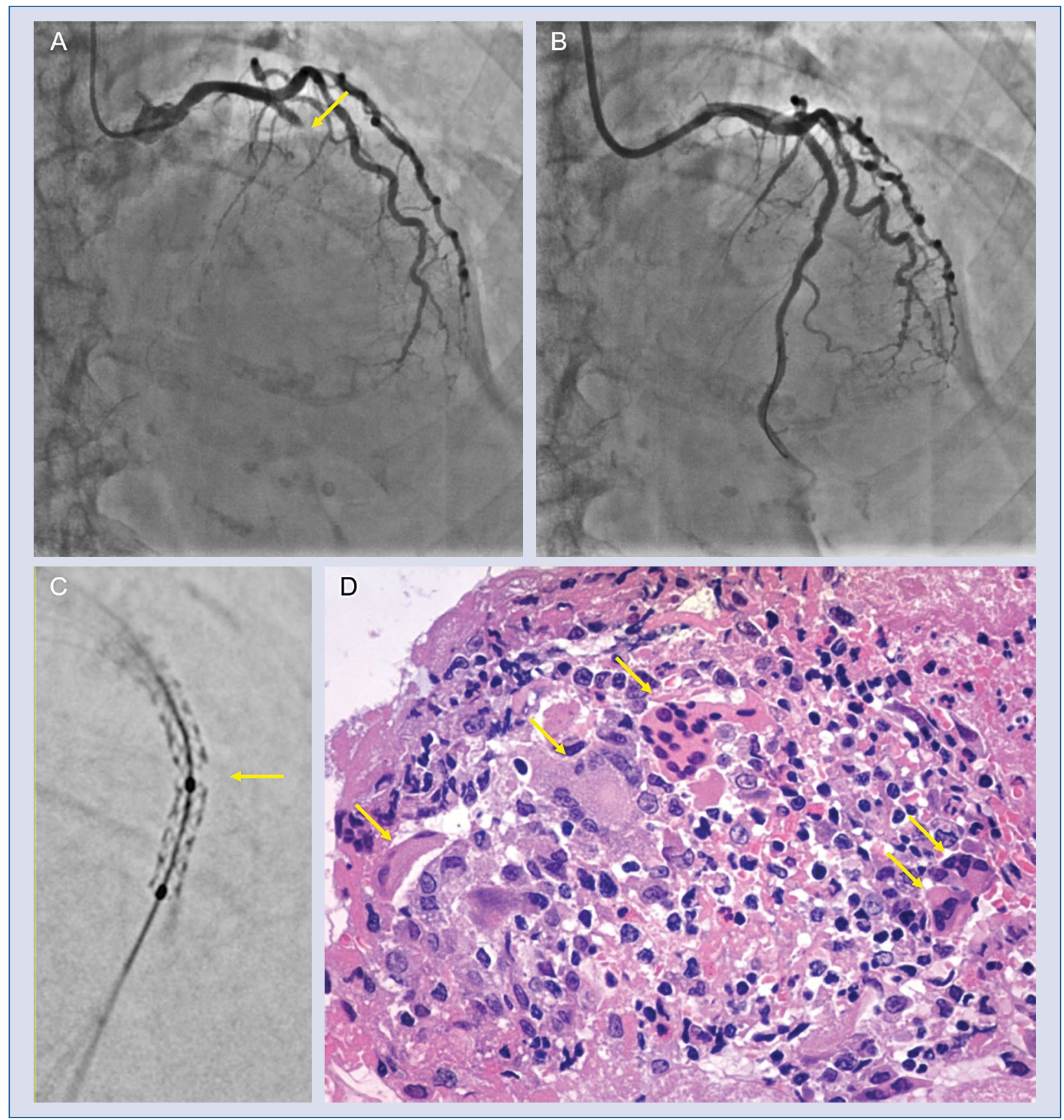

Figure 1. A. The initial coronary angiogram; B. The final coronary angiogram; C. The magnification image showing stent fracture; D. Histopathology. 